\title{
Malignant change in a chronic skin lesion induced by an intravenous cannula
}

\author{
Udim U Nkere, A Banjar
}

\begin{abstract}
Summary
A cutaneous wound subjected to continuous irritation has an increased potential for malignant degeneration. The types of trauma that may give rise to the initial injury are diverse and have been well documented. We report a case of one such lesion in a 65-year-old man who had a persistent right forearm wound for over three years. The wound arose from the site of venous cannula puncture. Malignant transformation occurred in a manner comparable to that seen in other chronic lesions (Marjolin's ulcer).
\end{abstract}

Keywords: venous cannula puncture, malignant transformation, skin lesion

Malignancy in a chronic wound is not uncommon and has been reported as occurring in chronic scars of diverse origin (box 1). ${ }^{1}$ Indeed, it has been pointed out that every cutaneous scar subjected to continuous irritation has an increased potential for malignant degeneration. ${ }^{1}$ Today the term 'Majolin's ulcer' is often applied to cutaneous lesions that have a malignancy within scar tissue. However, despite the diversity of causative factors, we have not found a Marjolin's ulcer in relation to a venous cannula site reported in the literature.

\section{Case history}

A 65-year-old man was admitted with a type 1 aortic dissection. He successfully underwent replacement of the ascending and arch of the aorta with re-implantation of the head and neck vessels into the aortic conduit. As part of the patient's resuscitative and peri-operative management an intravenous cannula was inserted into the cephalic vein of his right forearm. Four years later at the age of 68 , he

Department of

Cardiac Surgery,

Harefield Hospital,

Harefield, Middlesex

UB9 6JH, UK

UU Nkere

A Banjar

Correspondence to $\mathrm{Mr}$ Udim U Nkere, Department of Cardiothoracic Surgery,

Freeman Hospital, High

Heaton, Newcastle upon

Tyne NE7 7DN, UK

Accepted 7 March 1996

\begin{tabular}{|l|}
\hline $\begin{array}{l}\text { Warren's criteria for proving trauma } \\
\text { as causative in skin carcinoma }\end{array}$ \\
\hline - prior integrity of the tumour site \\
injury severe enough to initiate reparative \\
proliferation of cells \\
carcinoma arising after a reasonable latent \\
period \\
tumour type compatible with scar tissue and \\
with the anatomic location of the injury \\
\hline
\end{tabular}

Box 1 was admitted for further cardiac surgery (resection of a left ventricular aneurysm) and was found to have a raised $1-\mathrm{cm}$ diameter, erythaematous, fleshy lesion on the back of his right forearm (figure 1). There were no regional lymph nodes palpable. The lesion had started as a small 'scab' at the site of the intravenous cannula. $\mathrm{He}$ had suffered no previous skin disorder at this site nor at any other. Postoperatively, the scab had inadvertently been dislodged. Over the subsequent four years the wound received repeated inadvertent and self-inflicted (by 'picking') trauma. The resultant wound slowly enlarged. In the two years prior to his admission the wound had become pruritic. It periodically produced a foul-smelling discharge and would easily bleed. The patient would intermittently receive antibiotics from his general practitioner or treat himself with a 'plaster' over the wound. During his most recent admission an X-ray of the forearm showed no bony involvement and no evidence of a foreign body. Cultures of the discharge grew Staphylococcus aureus. After a week of antibiotic therapy a biopsy was taken from the lesion. Microscopic examination showed a superficial moderately differentiated squamous cell carcinoma (figure 2). The lesion was subsequently excised. The margins of the excised tumour were histologically clear of tumour. Sixteen months following the excision of the tumour the patient remains well and there is no evidence of recurrence or metastatic disease.

\section{Discussion}

A number of types of traumatic injury to the skin have been reported in the literature as giving rise to a wound that subsequently undergoes malignant degeneration. Warren's criteria $^{2}$ for proving trauma as causative in carcinoma of the skin (box 1) must be applied in order to limit the number claimed in the literature. We believe that the case presented here conforms with these criteria.

Because of the diversity of traumatic factors giving rise to chronic wounds and subsequent malignant degeneration, the overall incidence of Marjolin's ulcer is not known, neither is the precise mechanism of malignant degeneration. However, many theories have been proposed to explain how trauma to previously normal skin might lead to malignant transformation of the subsequent wound. It has been suggested that chronic suppuration, repetitive trauma and the age of the scar or ulcer are crucial 
Figure 1 A chronic, raised, 1-cm diameter, erythaematous, fleshy lesion on the back of the right forearm

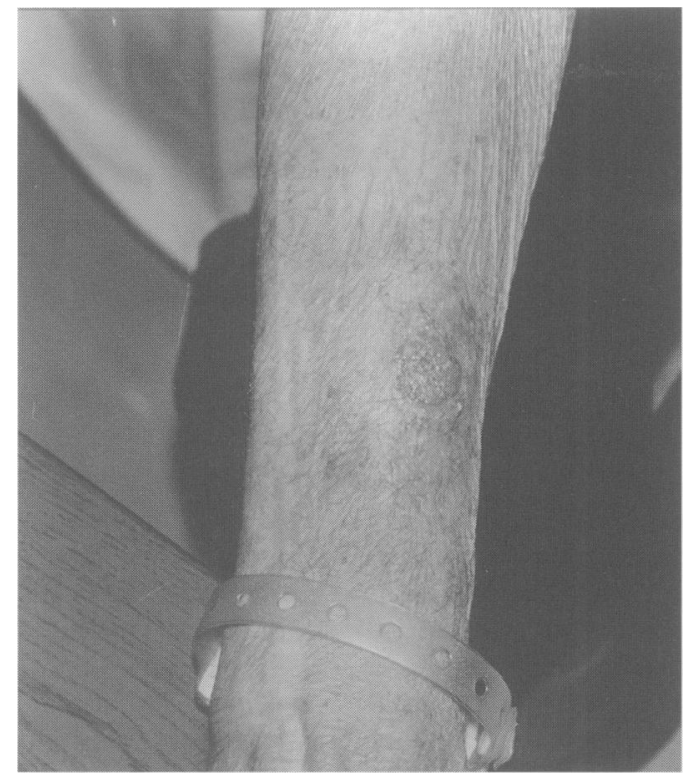

Figure 2 Epidermis showing severe cytological atypia and mitotic activity at all levels. In some areas (not shown) there was early invasion $(450 \times)$

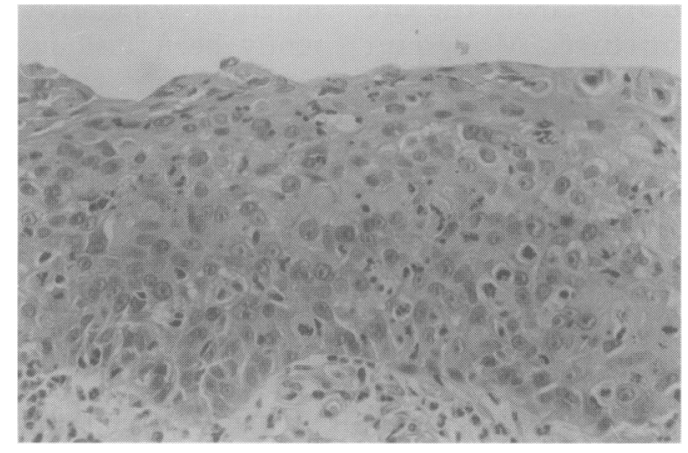

factors in malignant transformation. Indeed, an observation that may lend support to the theory that chronic irritation is a factor in the initiation of malignant transformation is that neoplasia are seen less often in burn scars that have been excised and resurfaced with a graft or flap. ${ }^{3}$ It would seem that the age of the ulcer, and therefore the longevity of its exposure to chronic irritation, is more important than the age of the patient. In particular, Treves ${ }^{4}$ reports an average lag period between ulcer and malignant degeneration of $\mathbf{3 2 . 5}$ years. It was Lawrence $^{5}$ who surmised that this lag period is longer in patients who were younger at the time of the injury. He proposed that the age of the skin and its previous environmental experience enhanced susceptibility to carcinogenic stimuli. This may help to explain why, in the case that we have presented, the lag period was only four years in a 65-year-old man.

It has been proposed that damaged and weakened epithelium is unable to withstand the effects of a carcinogen which may be produced by the wound. ${ }^{4}$ Even infection (which was evidently a factor in the case presented) acting on scar tissue or 'sensitised epithelium', may serve as a co-carcinogen stimulus, ie, a stimulus that of itself cannot produce a cancer but will accelerate, promote and bring to completion the appearance of a cancer in a cell in which the initiation of a cancer has been produced by a carcinogen.

Castillo and Goldsmith ${ }^{6}$ proposed that im- munologic depression produced by the relatively avascular scar predisposed to malignant degeneration of the lesion. The obliteration of scar tissue lymphatic drainage allows a delay in host immunological recognition. Furthermore, the poor vascularity hinders the blood-borne host defences from reaching target sights within the scar tissue.

As indicated by Glass and associates ${ }^{7}$ there is a tendency to manage cancerous ulcers as benign ulcers and a consequent failure to recognise the early development of malignancy in chronic skin lesions. Although the same symptoms may be ascribed to the presence of infection (as was the early assumption in the case presented), malignant transformation of a chronic ulcer may be heralded by an increase in pain, increasing discharge, foul odour, and bleeding. Increased suspicion of malignancy within a pressure sore should be aroused when the margins become verrucous. ${ }^{8}$

Biopsy remains the definitive diagnostic tool and should be undertaken in all chronically draining ulcers and tracts, especially those that have undergone recent change in appearance or amount of discharge. The biopsy should include the central area of the lesion as well as the margins and should be multiple sections deep enough to include the subcutaneous tissue. In certain selected cases, the role of fine needle aspiration cytology in the diagnosis of primary skin tumours has proved to be a sensitive and safe diagnostic method, being valuable both to the patient and to the preoperative planning of surgery. ${ }^{9}$ However, it is stressed by Daskalopoulou and associates ${ }^{9}$ that fine needle aspiration cytology cannot replace histology in the skin lesions, as there are a large number of tumours and tumour-like conditions in which tissue architecture is of paramount importance for accurate diagnosis.

Once biopsy has confirmed the presence of neoplasia, the lesion should be locally excised with histologically clear margins. The staging of the tumour has significant prognostic implications which may affect the overall surgical strategy and any adjuvant therapeutic intervention.

\begin{tabular}{l} 
Chronic scars that may give rise to a \\
Marjolin's ulcer \\
\hline - burns \\
- suprapubic cystostomy \\
- smallpox vaccination \\
- chronic hidradenitis suppurativa \\
- dessure sores \\
\hline
\end{tabular}

Box 2

\section{Learning point}

Suspect a Marjolin's ulcer in any posttraumatic lesion that does not heal after adequate treatment and a reasonable period of time 
The necessity of regional lymph node dissection is debated. Most authors would advocate such dissection only if there are clnically palpable nodes. ${ }^{3}$ However, regional lymph nodes may become enlarged as a result of chronic infection and/or metastases. Therefore, the size of the nodes is not a dependable indicator of the presence of malignancy. With the present data, the variation in opinion continues. However, all patients should at least receive local excision with careful follow-up over at least three years. If lymph enlargement occurs, lymph node dissection is indicated. For primary tumours larger than $4 \mathrm{~cm}$, Glass ${ }^{7}$ advocates node dissection at the time of primary surgery. In a series of patients studied by Ames in 1980, $98 \%$ of all recurrence were seen within three years from the time of primary excision. This included $92 \%$ of all regional lymph node metastases and $88 \%$ of

1 Berkwits L, Yarkony GM, Lewis V. Marjolin's ulcer complicating a pressure ulcer: case report and literature review. Arch Phys Med Rehabil 1986; 67: 831-3.

2 Warren S. Minimal criteria to prove causation of traumatic or occupational neoplasms. Ann Surg 1943; 117: 585.

3 Novick M, Gard DA, Hardy SB, Spira M. Burn scar carcinoma: a review and analysis of 46 cases. $\mathcal{f}$ Trauma 1977; 17: 809-17.

4 Treves N, Pack GT. Development of cancer in burn scars: analysis and report of thirty-four cases. Surg Gynecol Obstet 1930; 51: 749-82.

5 Lawrence EA. Carcinoma arising in scars of thermal burns: with special reference to the influence of age at burn on length of induction period. Surg Gynecol Obstet 1952; 95: 579-88. the distant metastases. The patient who survives three years without metastases has an excellent prognosis.

Various histological types of neoplasia are found in Marjolin's ulcer, including melanoma, osteogenic sarcoma, liposarcoma, adenocarcinoma, fibrosarcoma, carcinosarcoma, carcinoma in situ, basal cell carcinoma, and squamous cell carcinoma. ${ }^{4}$ This variation is perhaps an indication of the malignant potential that is within each layer of the damaged skin (epidermis, dermis and subcutaneous layer). Of these histological types, the most commonly encountered is the squamous cell carcinoma, followed by the basal cell carcinoma. ${ }^{4,5}$ However, the histological finding of pseudoepitheliomatous hyperplasia causes difficulty in distinguishing between benign and malignant. At times, this may be impossible. ${ }^{10}$

6 Castillo JL, Goldsmith HS. Burn scar malignancy in a possibly depressed immunologic setting. Surg Forum 1968; 19: $511-3$.

7 Glass RL, Spratt JS Jr, Perez-Mesa C. Fate of inadequately excised epidermoid carcinoma of skin. Surg Gynecol Obstet 1966; 122: $245-8$

8 Reuler JB, Cooney TG. Pressure sore: pathophysiology and Reuler JB, Cooney TG. Pressure sore: pathophysiology and
principles of management. Ann Intern Med 1981; 94: 661 6.

9 Daskalopoulou D, Maounis N, Kokalis G, Liodandonaki P, Belezini E, Markidou S. The role of fine needle aspiration cytology in the diagnosis of primary skin tumors. Arch Anat Cytol Pathol 1993; 41: 75-81.

10 White $C$, Weidman FD. Pseudoepitheliomatous hyperplasia at margins of cutaneous ulcers. $¥ A M A 1927 ; 88$ : 1959-63.

\title{
Carcinoma of the sigmoid colon presenting as a scrotal swelling
}

\author{
NP Bryan, A Jackson, AT Raftery
}

\section{Department of Surgery, Northern General Hospital, Sheffield S5, UK NP Bryan A Jackson AT Raftery}

Correspondence to Dr NP Bryan, 45 Brighton Terrace Road, Crookes, Sheffield S10 1NT, UK

Accepted 21 March 1996

\begin{abstract}
Summary
A case of adenocarcinoma of the sigmoid colon, presenting as a testicular mass, is described. At sigmoid colectomy widespread metastases were found and only palliative care could be offered thereafter. The incidence and age of such a presentation and manner of spread of the occult primary are discussed.
\end{abstract}

Keywords: testicular metastasis, colonic carcinoma, occult primary

Metastatic tumours of the testis are unusual; even rarer are tumours presenting in the testis from occult primaries. We present a case and review the literature.

\section{Case report}

A fit 75-year-old man presented with a swelling in the right scrotum. On examination there was an epididymal cyst and a nodule was palpated separate from the inferior pole of the right testis. He was otherwise asymptomatic.

An ultrasound scan confirmed a solid mass ( $2 \mathrm{~cm}$ diameter), suspicious of malignancy, inferior to the right testicle. At orchidectomy a firm 2-cm nodule was found adjacent to the epididymal cyst (figure). It was external to the tunica vaginalis. There was a hydrocele of the cord and testis but no macroscopic patent processus vaginalis, although a microscopic connection with the peritoneal cavity could not be excluded. Histology confirmed the presence of a moderately differentiated adenocarcinoma. Tumour markers were positive for 